\title{
Cáncer de páncreas: de las lesiones premalignas a la paliación
}

\author{
Félix Ignacio Téllez-Ávila* \\ Departamento de Endoscopia Gastrointestinal, Instituto Nacional de Ciencias Médicas y Nutrición Salvador Zubirán, Ciudad de México, México
}

\section{Resumen}

En el presente trabajo se comentan los estudios más relevantes, a consideración del autor, de los trabajos mostrados en relación a cáncer de páncreas desde el tamizaje, hasta la paliación pasando por el tratamiento de la enfermedad. El cáncer de páncreas es el número 11 en frecuencia considerando todos los cánceres y el 3ro como causa de muerte. El riesgo anual para población general es de 1.6\%, y en el 2018 en estados unidos se diagnosticaron 55,400 nuevos casos y se registraron 44, 330 muertes. Se ha reportado que tiene un aumento en frecuencia de $0.5 \%$ anual y se considera que sea la principal causa de muerte por cáncer para el año 2030. Actualmente la tasa de supervivencia a 5 años es de solo el $8 \%$ ya que el $80 \%$ de los casos se diagnostican en estadios avanzados. Actualmente no hay ningún biomarcador para el screening o para el diagnóstico y únicamente conocemos algunos factores de riesgo.

\section{Tamizaje}

Antes que nada mencionar que no se presentaron trabajos al respecto. Se conocen algunas lesiones premalignas para el cáncer de páncreas como son la neoplasia intraepitelial pancreática (PanIN), la neoplasia mucinosa papilar intraductal (IPMN) y las neoplasias quísticas mucinosas (NQM). Específicamente estas lesiones tienen guías de tratamiento y a grandes rasgos cuando se detecta displasia de alto grado se recomienda tratamiento quirúrgico y en los casos de displasia de bajo grado solo seguimiento.

A pesar de estos avances, en la población general aún no se ha logrado encontrar una herramienta de tamizaje que sea factible y costo beneficio. De las lesiones premalignas, las NQM y las IPMNs son susceptibles a detectarse por medios de imagen, sin embargo, la PanIN es una lesión plana.

Actualmente el tamizaje solo está indicado en aquellos pacientes con antecedentes heredofamiliares importantes ${ }^{1} \mathrm{o}$ con alteraciones genéticas asociadas con el desarrollo de cáncer de páncreas ${ }^{1}$. Sin embargo, no existen estudios que sustenten el tamizaje. Existen varias interrogantes al respecto como ¿cuál es el mejor método de tamizaje para estos pacientes?, ¿el tamizaje ayuda a prevenir casos de cáncer en pacientes de alto riesgo?, ¿realizar el tamizaje es costo-benéfico? En relación a cuál es el mejor método de imagen para tamizaje parece que el ultrasonido endoscópico es la mejor opción incluyendo la ventaja de poder tomar muestras de tejido para la valoración por patología, aunque existen muy pocos datos para apoyar dicha afirmación. En relación a si ayuda a prevenir casos de cáncer existen pocos datos pero al parecer se requiere realizar el tamizaje en 1000 pacientes de alto riesgo para captar 7.8 casos (IC95\% 3.6-14.7) y en el caso de realizar seguimiento y no una sola medición, se llegan a detectar hasta 15.6 casos por cada 1000 pacientes (IC95\% 9.3-24.5) $)^{1}$.

Por lo anterior, hasta el momento no existe un método aceptado o recomendado y todo se basa en protocolos locales de hospitales que cuentan con servicios o clínicas dedicados a esta patología.

\section{Correspondencia:}




\section{Paliación}

En un estudio de Ramireddy S, et al. (Tu1353) ${ }^{2}$ en el cual se ofreció radiofrecuencia guiada por USE (USE RFA) a 10 pacientes con cáncer no resecable se pudo observar que en 6/10 pacientes la lesión presentó disminución del tamaño, 2/10 el tumor progresó y 2 pacientes no tuvieron seguimiento. La mediana de supervivencia fue de 17 (IQR 16-26) meses y no se reportaron complicaciones. Por lo anterior los autores concluyeron que la USE RFA pudiera ser considerada como un método adyuvante a la quimioterapia en pacientes con esta enfermedad. Otro estudio similar de China $(T u 1357)^{3}$ en 8 pacientes mostró resultados en la misma dirección, y un estudio de Dancour A, et al. (Tu1390) ${ }^{4}$ mostro resultados similares con la particularidad de que en este estudio se incluyeron datos sobre USE RFA en metástasis.

Aunque no es propiamente paliación un estudio muy interesante fue presentado por Rustgi S, et al. (Tu1387) ${ }^{5}$ en el cual se revisaron bases de datos de pacientes con cáncer de páncreas en todos los estadios entre el 2000-2011 encontrando 32,510 casos de los cuales solo a $7094(21.8 \%)$ se les había realizado un USE encontrando que la supervivencia de los pacientes que se sometieron a USE fue mayor (10 vs 5.5 meses; $\mathrm{p}=0.01$ ) con un hazard ratio de 0.73 (IC95\%: 0.720.75). La proporción de pacientes que se sometieron a un USE se incrementó en el tiempo: $10.9 \%$ entre 2000 $2003,21.2 \%$ entre $2004-2007$ y $32.8 \%$ entre $2008-2011$, $\mathrm{p}=0.01$. Además, se observó que el USE se utilizó más en aquellos pacientes con mayor edad y con mayor índice de Charlson. Por lo cual los autores concluyeron que el uso del USE en pacientes con cáncer de páncreas, en cualquier estadio de la enfermedad tiene un impacto favorable en la supervivencia de los pacientes a pesar de que este se utiliza con mayor frecuencia en pacientes de mayor edad y con mayor número de comorbilidades.

\section{Tratamiento}

Sin duda un punto importante de innovación estuvo en este rubro. Por años el tratamiento del cáncer de páncreas se ha mantenido prácticamente estático y se cree que en parte es relacionado a que el adenocarcinoma de páncreas es una enfermedad sistémica (no solo localizada al páncreas) y a que existen varios "tipos" de adenocarcinoma y que aún no podemos discernir entre ellos, esto explicaría porque la misma quimioterapia no es efectiva en todos los pacientes. Por lo anterior, al igual que en otros tumores, el tratamiento para el cáncer de páncreas seguramente tenderá a individualizarse y para lograr esto los estudios que menciono a continuación, tal vez, tengan un importante papel en algunos años.

Wu C, et al. (Mo1368) ${ }^{6}$ presentó su trabajo de cultivo celular a partir de muestras de tejido de cánceres de páncreas por medio de biopsias guiadas por USE y los comparó con aquellos realizadas a partir de muestras de tejido obtenido por cirugía. Lo que encontró es que ambos métodos son útiles para desarrollar crecimiento de células tumorales ex vivo. En un estudio en el mismo sentido Cho $\mathrm{CM}$, et al. $(387)^{7}$ estudiaron si un modelo 3D de cultivo de células tumorales de cáncer de páncreas tomadas por biopsias guiadas por USE eran útiles. Se utilizaron muestras de 16 pacientes en los cuales se tomaron biopsias (no aspirados) de tumores pancreáticos. Los resultados fueron prometedores en relación al desarrollo de células tumorales y abre la puerta, al igual que el estudio de Wu, et al. para crear modelos para pruebas in vitro de susceptibilidad a quimioterapia.

\section{Conflicto de intereses}

No existen conflictos de interés. Sin patrocinio de la industria.

\section{Bibliografía}

1. Guda N. Screening for pancreatic cancer by EUS and genetic testing. DDW; 2019 mayo 17-21; San Diego, CA

2. Ramireddy S, Cen P, Rowe J, et al. EUS RFA likely offers survival benefits in patients with unresectable pancreatic cancer: results from a single center study with long term follow-up. DDW; 2019 mayo 17-21; San Diego, CA, Tu1353.

3. Yang J, Zhang X. Feasibility and safety of EUS-guided radiofrequency ablation in unresectable pancreatic cancer. DDW; 2019 mayo 17-21; San Diego, CA, Tu1357.

4. Dancour A, Rottenstreich M, Sheynkman G, et al. EUS-guided, RFA ablation of benign and malignant pancreatic neoplasms and extrapancreatic metastasis is feasible and safe. DDW; 2019 mayo 17-21; San Diego, CA, Tu1390.

5. Rustgi S, Amin S, Yang A, et al. Improved survival with EUS for all stages of pancreatic cancer: a propensity score analysis. DDW; 2019 mayo 17-21; San Diego, CA, Tu1387.

6. Wu C, Lim JS, Lam S, et al. Successful growth of recombinant co-cultured pancreatic ductal adenocarcinoma tumoroids via EUS-guided FNB specimens for individualized cancer therapy. DDW; 2019 mayo 17-21; San Diego, CA, Mo1368.

7. Cho CM, Nam SY, Bae HI, Seo AN. Feasibility of a three-dimensional spheroid culture using endoscopic ultrasound tissue sampling in pancreatic cancer. DDW; 2019 mayo 17-21; San Diego, CA, 387. 